\title{
Liquid metal batteries and their magnetohydrodynamic instabilities
}

\section{Metal pad instabilities in liquid metal batteries}

Authors: Oleg Zikanov

Phys. Rev. E 92, 063021 (2015)

\section{Fluid Mechanics of Liquid Metal Batteries}

Authors: Douglas H. Kelley and Tom Weier

Appl. Mech. Rev. 70(2): 020801 (2018)

\section{Recommended with a Commentary by Brian Skinner, Ohio State University}

Liquid metals are an old source of fascination in condensed matter physics. For example, understanding their electrical conductivity (even its order of magnitude) was a serious puzzle for many decades, since one cannot use Bloch's theorem in a setting with no periodic structure. (This puzzle was finally cracked in the 1960 s - Ref. [1] by Ziman gives a very enjoyable overview.) Recent years have seen a mild revival of interest in liquid metals, with a focus on their mechanical properties [2]. For example, the surface tension of many liquid metals is altered enormously by the formation of an oxide layer on its surface, which presumably grows by the Cabrera-Mott mechanism [3]. (Briefly: an electron first quantumtunnels across the oxide layer from the bulk metal to bind to an oxygen atom on the surface, and then drags a metal ion across the oxide via the Coulomb attraction, so that the oxide thickness is tunneling-limited and grows logarithmically in time.) Consequently, the metal's surface tension can be strongly altered (and even brought to zero) by an applied voltage; Ref. [4] presents some fun experiments in which a spherical liquid metal droplet is reversibly turned into fractal shapes.

The two recommended papers illustrate yet another reason why condensed matter physicists might consider reviving their interest in liquid metals. They concern the operation of a certain new type of battery made from liquid metals. The usefulness of these batteries turns out to be limited by a surprising magnetohydrodynamic instability.

Before describing these papers, it is worth remarking on the general context that is driving research into new types of battery. The cost of energy generation through renewable sources (and solar panels in particular) has fallen so dramatically in the last decade that this cost is no longer the primary bottleneck for achieving an all-renewable energy grid [5]. A more significant bottleneck now is the cost and efficiency of energy storage. Efficient, large-scale energy storage is needed over a wide range of time scales, from seconds to months, in order 
to even out the differences between power supply (which depends, for example, on when the sun is shining) and user demand, and thereby to prevent blackouts.

For energy storage on the critical time scale of 2-6 hours, the dominant technology at present is lithium-ion batteries. Li-ion batteries operate by a process of reversible intercalation of $\mathrm{Li}^{+}$ions into graphite. During the process of charging the battery, $\mathrm{Li}^{+}$ions are driven out of a Li-rich metal and attracted by an applied voltage to nestle between the graphene sheets that comprise a bulk graphite electrode (see, e.g., Ref. [6] for a textbook-level overview).

At a theoretical level, understanding this charging process well enough to predict and improve upon the performance of Li-ion batteries is a messy business. It requires one not just to understand the electrochemistry involved, but also to think about the spatial correlations between intercalated Li ions, which are mediated by (screened) Coulomb interactions and elastic stresses in the graphite. The kinetics of the charging process are determined by the process of ion diffusion, both between the sheets of the graphite and through the electrolyte layer that separates the anode and cathode of the battery, which is similarly complicated to understand.

Li-ion batteries also suffer from several practical limitations. Most notably, they degrade over time due to cracking and other changes of the microstructure. Such changes are a seemingly inevitable consequence of the repeated expansion and contraction of the graphite during charging (intercalation) and discharging (de-intercalation). Their maximum power is also limited by the slow ion diffusion, as mentioned above.

Because of these limitations, there is intense research into alternative battery technologies [7]. One especially interesting competitor is the liquid metal battery, in which both the anode and cathode are made from a liquid metal rather than from any solid material. A schematic is shown in Fig. 1. The idea is that the two metals naturally self-segregate on opposite sides of a molten salt electrolyte because of their different densities. During the discharging process, a metal ion diffuses from the anode across the electrolyte to form a (lower-energy) liquid metal alloy on the cathode side. The charging process re-segregates the two metals.
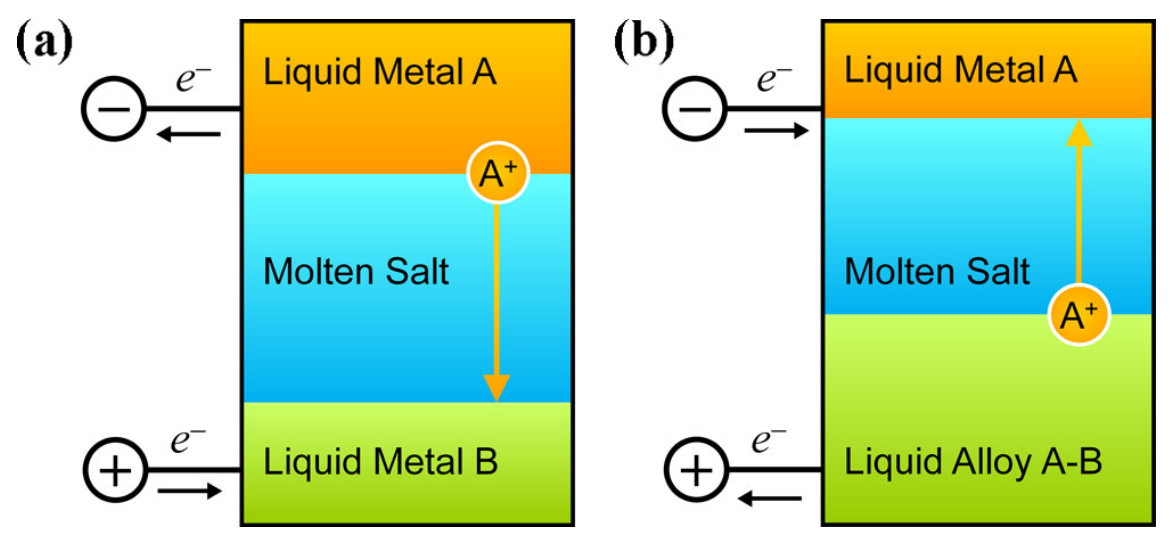

Figure 1: Schematic of a liquid metal battery during (a) discharging (b) charging. Figure from Ref. [7].

This liquid metal design offers a few tantalizing advantages. First, it offers the promise of much faster kinetics, and therefore higher power, since everything is in the liquid phase 
and there is no difficult intercalation process. Second, a liquid metal battery is immune to degradation by cracking or other microstructure changes, since there is no solid structure at all. Thus one can hope for both more longevity and more theoretical simplicity.

There is, however, one glaring disadvantage (in addition to the relatively limited set of metals that are liquids near room temperature). If the battery is jostled with sufficient force, and if the electrolyte layer isn't very thick, then the two liquid metals might come into contact with each other. Such an event would short-circuit the battery and potentially start a fire.

Still, one could think that as long as the battery is used in a sedentary application, then it should be safe. The two recommended papers show that, in fact, liquid metal batteries can be intrinsically unstable due to magnetohydrodynamic effects. In other words, when a large current flows through the battery, the resulting Lorentz forces can drive the two metals to slosh violently and potentially short-circuit.

The first recommended paper by Zikanov focuses on a particular type of instability, called a "metal pad instability" by the author (due to its close analog with a similar instability that plagues the industrial process of refining aluminum). This instability is driven by a stray magnetic field in the vertical direction; such stray fields are often present due to the electric current coming in from the battery's supply lines. If the electric current in the interior of the battery runs purely in the vertical direction, then such a magnetic field produces no Lorentz force. But if the liquid metals are perturbed a bit, so that the distance between them varies across the horizontal direction, then the electric current becomes focused near their point of closest contact and horizontal currents arise within the liquid metals. These horizontal currents produce a Lorentz force on the fluid in the perpendicular inplane direction (see Fig. 2). The result is that an initially small tilting of the fluid can be amplified into a clockwise or counterclockwise sloshing rotation of the fluid, like wine being swirled in a glass.

Zikanov describes this instability quantitatively using a simple model in which the liquid metal is approximated by a rigid metal plate, suspended like a physical pendulum by gravity. (This approximation is reasonable since the dominant instability mode corresponds to the longest wavelength of fluid motion, i.e.

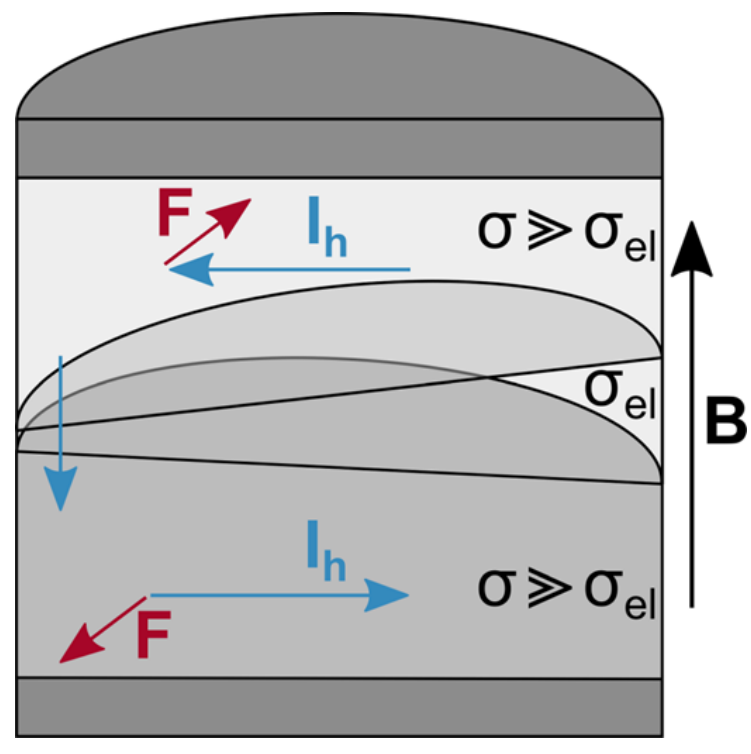

Figure 2: Schematic illustration of the metal pad instability in a liquid metal battery (taken from the second recommended paper). $\sigma$ and $\sigma_{e l}$ denote the electrical conductivity of the metals and the electrolyte layer, respectively. The Lorentz forces $F$ point into the page (in the upper layer) and out of the page (in the lower layer). to the horizontal size of the battery.) By analyzing the equation of motion the author derives a simple criterion for instability in terms of the strength of the current, the strength of the stray magnetic field, and geometric factors. $\mathrm{Nu}-$ merical estimate suggest that such instabilities are within the plausible operating range of real liquid metal batteries. Interestingly, within Zikonov's model the instability seems un- 
avoidable in situations where the battery has a square or circular cross section (and one neglects viscous damping). In such cases the instability seems to arise from a degeneracy between two transverse fluid oscillation modes. Indeed, a more recent work [8] showed that for a battery with rectangular cross section there is actually a dense set of such instability points, such the fluid is easily rendered unstable any time the aspect ratio is equal to $\sqrt{m / n}$, where $m$ and $n$ are odd numbers. The instability is dampened when the fluid has a finite viscosity, but when this viscosity is not too large the threshold value of the magnetic field or current density that produces the instability retains a jagged dependence on the aspect ratio.

What's more, it turns out that this "metal pad instability" is not the only type of fluid instability that can appear in liquid metal batteries. In the second recommended paper, Kelley and Weier present a very nice overview of magnetohydrodynamic instabilities in liquid metal batteries, including the one considered by Zikonov. These additional instabilities include one arising from Marangoni flow - in which a gradient of temperature produces a gradient of surface tension, which can strongly drive fluid flow - and a Tayler instability - in which the intense magnetic pressure arising from a vertical current can destabilize the fluid and cause its flow to "kink". (The latter has a significant research history in both plasma physics and astrophysics, see, e.g., [9] and the references therein.) For readers with even a passing interest in fluid mechanics, this paper constitutes an enjoyable overview of a wealth of different physical phenomena.

It is unusual to find a research topic that makes connections to such a broad swath of physics concepts and subfields. More unusual still that the topic should have direct relevance for a problem as pressing as energy storage. Whether these factors will be enough to drive a renaissance of interest in liquid metals among condensed matter physicists remains to be seen.

\section{References}

[1] J. M. Ziman, "Review Lecture - Electrons in liquid metals and other disordered systems," Proc. R. Soc. Lond. A318401-420 (1970).

[2] M. Dickey, "Liquid metals at room temperature," Physics Today 74, 4, 30 (2021).

[3] N. Cabrera and N. F. Mott, "Theory of the oxidation of metals," Rep. Prog. Phys. 12 163 (1949).

[4] Minyung Song, Karen E. Daniels, Abolfazl Kiani, Sahar Rashid-Nadimi, and Michael D. Dickey, "Interfacial Tension Modulation of Liquid Metal via Electrochemical Oxidation," Adv. Intell. Syst., 3: 2100024 (2021).

[5] David Kramer, "Better ways to store energy are needed to attain Biden's carbon-free grid," Physics Today 74, 9, 20 (2021).

[6] Gholam-Abbas Nazri and Gianfranci Pistoia, Eds., Lithium Batteries: Science and Technology, NY: Springer (2003). 
[7] Hojong Kim et. al., "Liquid Metal Batteries: Past, Present, and Future," Chem. Rev. 2013, 113, 3, 2075-2099 (2012).

[8] G. Politis and J. Priede, "Fractality of metal pad instability threshold in rectangular cells." Journal of Fluid Mechanics, 915, A101 (2021).

[9] Martin Seilmayer et. al., "Experimental Evidence for a Transient Tayler Instability in a Cylindrical Liquid-Metal Column," Phys. Rev. Lett. 108, 244501 (2012). 